\title{
Provision of Adolescent-Friendly Sexual and Reproductive Health (SRH) Services Integrated into HIV Treatment and Care
}

\author{
$\mathrm{Ng}{ }^{` a m b i ~ K}$
}

Pride Community Health Organization, Bachelor of Social Work, Kafue District, Republic of Zambia

*Corresponding author: Ng'ambi K, Pride Community Health Organization, Bachelor of Social Work, P.O.Box 360297, Luangwa Drive, Kafue House, Kafue District, Republic of Zambia, Tel: +260 9774211 64, E-mail: Masausokenanngambi@Yahoo.Com

Citation: Ng ambi K (2016) Provision of Adolescent-Friendly Sexual and Reproductive Health (SRH) Services Integrated into HIV Treatment and Care. J Aids Hiv Infec 2(2): 203. doi: 10.15744/2454-499X.2.203

Received Date: October 03, 2016 Accepted Date: November 11, 2016 Published Date: November 14, 2016

\begin{abstract}
Background: Integration of HIV prevention services into reproductive health, care support and treatment programs can potentially increase the uptake of FP, dual protection and reduce vertical transmission of HIV. Pride Community Health Organization supports Adolescent Sexual Reproductive Health Services targeting young people in the age group of 10 - 19 and 20 - 24 years. An average of 65 young people each month access information and services in the -One Stop Shop including Peer Supporter Program for Adolescents and Children Living with HIV to improve HIV treatment and care outcomes for peers.

Methods: To improve access to, and uptake of Adolescent Sexual Reproductive Health Services, HIV counseling and Testing, FP and referrals were integrated in the counselling process at the Youth Friendly Corner site with specific emphasis on dual protection and condom use. Peer Educators in the age group of $10-19$ and 20- 24 girls and boys implemented sexual and reproductive health programs at Estates Clinic which did not have youth friendly services at that time. A room was secured to be used for provision of Adolescent - Youth Friendly sexual and reproductive health services at Estate Clinic. Peer educators disseminate HIV and AIDS information and refer young people for services to the ART and TB nurses who are permanently located within the -One Stop Shop. Peer educators also conduct community awareness meetings in schools, bars and taverns.

Results: From January 2015 to December, 2015: 889 adolescents received HIV and AIDS information and 441 were tested for HIV, and 40 clients were referred for ART services. A total of 47 were also referred for STI screening, and 101 were referred for Voluntary Medical Male Circumcision. A total of 17,238 condoms were distributed at the -One Stop Shop.

Conclusions: One Stop Shop provides a friendly space where young people can access information and increases uptake to ASRH services. Adolescents peer educators create demand for adherence and retention support, mitigation of stigma and discrimination. Health care providers work is easier and waiting periods reduced. Adolescent's specific interventions increase access SRH and HIV services.
\end{abstract}

Keywords: Adolescent - Friendly SRH Services; Integration; HIV treatment and Care

\section{Introduction}

The rapid scale up of antiretroviral treatment (ART) and HIV care across Africa over the past five years has provoked an important and lively debate about the merits and the impact of harnessing community support in advancing the continuum of prevention, treatment and care in relation to effective collaboration. The major decreases in international funding designated for HIV/AIDS programmes from the US President's Emergency Plan for AIDS Relief, the Global Fund to Fight AIDS, Tuberculosis and Malaria, and a range of other donors has raised concerns about how reduction in funding could impact existing services [1,2].

On the other hand, some have argued that the reduction funding for HIV offers an opportunity to leverage scarce resources. It is still common in Africa to see Clinics and community-based organizations (CBO's) continue to operate in silos due to limited structural mechanisms, opportunities and or entry points that effectively facilitate, coordinate and support meaningful engagement between them. Health facilities and their staff resources are often overextended and lack the capacity and skills to engage and integrate communities within health service planning and delivery. From a community perspective, many CBOs have limited technical and organizational capacity (which is compounded when they are excluded from health service decision-making and programme planning), contributing to a power differential that can often lead to a conflicting versus complementary relationship.

The pervasive impact at the frontline of service provision is that community is not understood or acknowledged as a critical and equal partner by health facilities. Although CBO's have value to add around demand generation, adherence and retention support, 
mitigation of stigma and discrimination, and design and implementation of innovative community-based service delivery models, they continue to experience isolation and segregation rather than clinic-CBO collaboration, co-ordination and co-operation. There is sufficient evidence that community based support has helped in prevention and also provision of care for HIV and other diseases in Southern Africa [3]. Community Health Workers play an important role in supporting retention in care through defaulter tracing, adherence counseling and collection of drugs from service points. There are many community based support models but the most notable ones are adherence support to ART by CHWs, either during home visits or through mobile phone reminders. This type of a model increases adherence support within community settings. Recent studies have indicated that the support of close relatives is fundamental in coping with HIV/AIDS and in accessing the emotional and material support necessary for sustained adherence to treatment [4].

Equally important is that practitioners on the ground can increasingly recognize that quality HIV care cannot be provided without improving access to, and uptake of Adolescent Sexual Reproductive Health Services, HIV counselling and Testing, FP and referrals. The Zambian experience with integrating adolescent - friendly sexual and reproductive health services into HIV treatment and care, described in this paper, provides evidence that $\mathrm{CBO} /$ Clinic collaboration strategy can simultaneously strengthen both HIV/ AIDS services and the broader health system in which those services are embedded. This approach ensures joint health system and community ownership and participation in planning and execution of activities, and builds operational bridges between the clinic and the $\mathrm{CBO}$. Clinic/community models should ensure that power differentials are minimized through ongoing support and jointly-established goals requiring the full participation of both clinic and community. Community support can contribute considerably reducing death rates and produce better overall ART outcomes. Communities could largely play an important contributory role in countries desperately trying to scale up ART with limited resources [5].

\section{Materials and Methods}

To improve access to, and uptake of Adolescent Sexual Reproductive Health Services, HIV counselling and Testing, FP and referrals, with specific emphasis on dual protection and condom use were integrated in the counselling process at the Youth Friendly Corner site. Youth - friendly services are services that all adolescents and young people are able to obtain, and these services must meet adolescents' expectations and needs and improve their health.

Peer Educators in the age group of $10-19$ and 20 - 24 girls and boys implemented sexual and reproductive health programs at Estates Clinic which did not have Youth Friendly Services at that time. The number of Peer Educators who implemented sexual and reproductive health programs was 15 (4 adolescent male and 4 adolescent girls: 15 - 19 years) and 3 young men and 4 young girls).

With support of Pride Community Health Organization and other partners, in collaboration with the Kafue District Medical Office, secured a room to be used for provision of Adolescent - Youth Friendly sexual and Reproductive Health Services integrated into HIV treatment and care. Peer educators were trained by the Kafue District Medical Office and assigned to disseminate HIV and AIDS information and refer young people for services. Some of issues that were discussed by their peers in these sessions were adherence counseling, contraceptive counseling, safer sex practices, and family support systems. Peer counselors play a very important role by acting as role models, raising awareness, and being visible in the community [6]. The level of education for most of these educators who were implementing this project was secondary school. Human resource shortage has placed a heavy burden on the few health care workers available at designated health facilities in our country [7].

ART and TB nurses provide services within the center reducing the long process of adolescents passing through different departments. Peer educators also conduct community awareness meetings in schools and other Hotspot sites such as bars and taverns and conduct home follow-ups to adolescents who miss their clinic appointments.

\section{The Setting}

The one-stop shop is a separate two-room structure for adolescent services, and is based at the Kafue Estates Health Facility which is located in Kafue Estates community 5 of Kafue district. The health facility caters for urban and peri-urban areas around the catchment zone. One room is designated as a private consultation room, and the other a communal information room for group meetings and SRH information dissemination (including a television and information, education and communication materials). The space is decorated in color and intended to be appealing to adolescents. Two dedicated nurses have been are assigned specifically to the provision of adolescent-friendly SRH services in the space.

\section{Results}

From January 2015 to December, 2015: 889 adolescents boys, girls, young men and women 15 - 24 years were reached with HIV and AIDS information and 441 were referred and successfully tested for HIV. A total of 40 adolescent girls, boys, young men and women who tested HIV positive were put on Antiviral vital treatment. (14 boys, 10 young men, 16 girls and 10 young women). 47 adolescents and young people ( 21 girls and women and 26 boys and men) were also referred for STI screening, and 101 were referred for Voluntary Medical Male Circumcision. Peer Educators also distributed a total of 17, 238 condoms at the Adolescent Youth Friendly "One Stop Shop" [8,9] (Figure 1). 
Access to Services

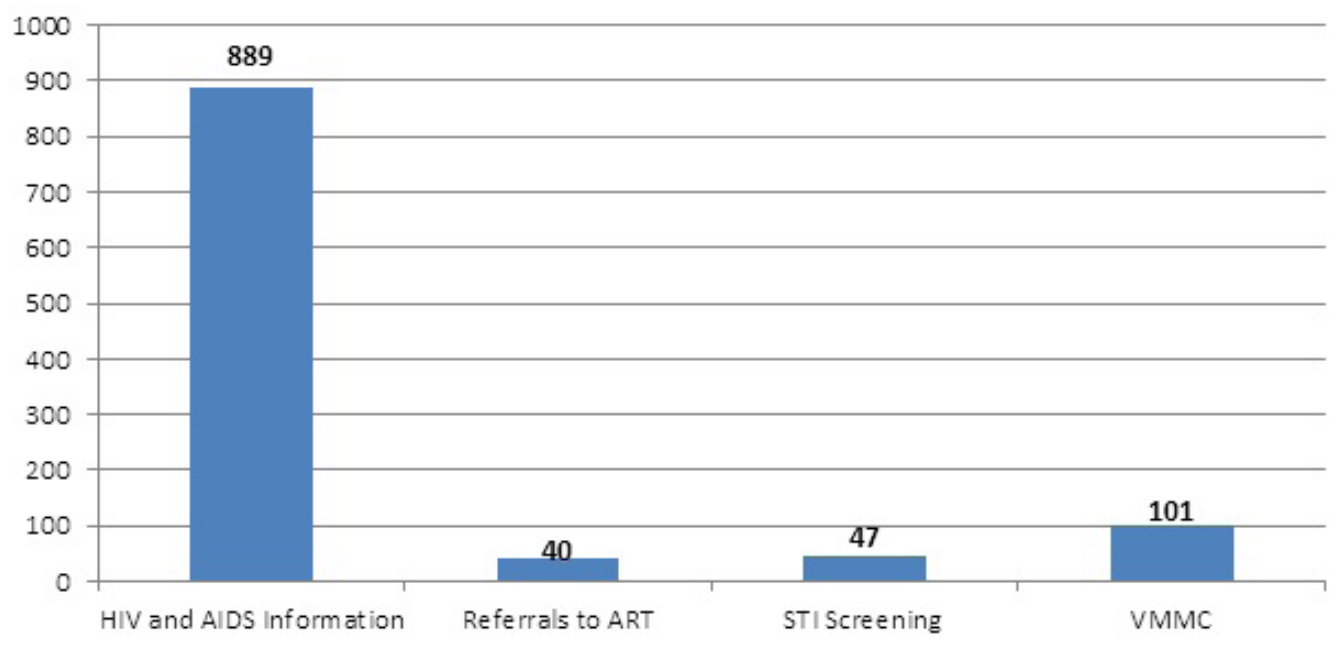

Figure 1: HIV and AIDS information from January 2015 to December 2015

\section{Discussions}

Adolescents accessing the one-stop shop benefited from improved, Retention in care; access to an increased range of adolescentfriendly SRH services; environment and separate space to meet, share and access services; knowledge of SRH, HIV and adherence; health outcomes, including virological suppression; household members tested for HIV; stigma-free environment and improved health provider attitudes; and psychosocial support.

The clinic and $\mathrm{CBO}$ benefited from: dedicated resources to reach and serve adolescents; increased insight and sensitivity to the lived experiences of their adolescent patients; increased numbers of adolescents accessing and retained in services; improved adolescent health outcomes; a formal agreement and working relationship between the clinic and CBO; improved clinic and CBO capacity to reach and return adolescents who had been lost to care; increased caregiver engagement and disclosure to adolescents; and more positive health provider attitudes.

The sample of this study were adolescents 15 - 24 years, and the criteria used was because adolescents and young people represent a significant demographic and socio - economic force, and are a major factor in influencing public health trends. They are highly affected by various health problems, including communicable and non- communicable diseases such as STI's, including HIV and AIDS, and behaviour related problems. Adolescent girls 15 - 19 years account for $4.9 \%$ of new HIV infections in Zambia (ZDHS 2013/14) [8]. However, often adolescent girls most affected by HIV are unable to access HIV and sexual and reproductive health (SRH) services including family planning, sexually transmitted infection (STI) screening and treatment mainly because of HIV related stigma and discrimination.

Some of the limitations which were encountered were; the sample group in the research study was limited to a small number of adolescents' living with HIV, therefore, the findings may be an unrealistic representation of the entire adolescents' population. Our programme hoped to have an equal number of both boys' and girls" adolescents' living with HIV answering the questionnaires, unfortunately however, due to the exceedingly higher percentage of girls" adolescents' living with HIV, in comparison to male adolescents' living with HIV, and accessing ART services at the facility, these expectations were unattainable. Another limitation was due to the personal nature of the research there was no guarantee that the participants were completely honest when answering all the questions.

There are of course, ongoing challenges to successful integration. The challenges of adolescent ART adherence and SRH are complex and situated within the contexts in which adolescents live. Many factors outside of the intervention site, such as the home and community, impact adolescent health. Child abuse, rape, gender-based violence, forced marriages and HIV stigma are barriers to adolescent SRH and service access. These challenges are compounded by multiple additional stressors such as poverty and unemployment, and impact care giving and household resilience [10,11].

Workforce shortages continue to be the single greatest challenge to scale up, and adding HIV care tasks to overburdened staff may raise concerns about quality [12]. Facility based training for health staff can be useful to support more efficient human resources management, but further evaluation is necessary to measure quality improvement. It is hoped that integration will bring additional efficiencies overall that can help mitigate the effects of human resource constraints on quality and sustainability of services [13]. The use of HIV-focused funding to increase numbers in the overall health workforce is increasingly essential to scale up success. 


\section{Conclusion}

Despite challenges associated with adolescents' own HIV status, environments that facilitate this process exist and can be strengthened. Promoting the "One Stop Shop" concept requires in-depth and context-specific understanding of the factors that enable and undermine this process. Limitations in this understanding may lead to bottlenecks in the successful design and implementation of this innovative community-based service delivery model.

According to Estates Clinic the significance of having this "One Stop Shop" has provided not only the friendly space where young people can access information, but has also increased uptake to ASRH services. The adolescents peer educators have added value to demand generation, adherence and retention support, mitigation of stigma and discrimination. The establishment of the One Stop Shop has made the work of the health care providers easier and reduced on the long waiting periods and separated adolescents sitting amongst adults where they felt stigmatized and created reluctance amongst adolescents to access SRH and HIV services in Kafue.

\section{Acknowledgment}

The intervention was supported by the Southern Africa AIDS Trust Zambia, and Paediatric AIDS Treatment for Africa. The author also wishes to acknowledge the support of the Ministry of Health and the Kafue District Health Office in Lusaka Province.

\section{Supplymentary Information}

\section{References}

1. Lima VD, Johnston K, Hogg RS, Levy AR, Harrigan PR, et al. (2008) Expanded access to highly active antiretroviral therapy: a potentially powerful strategy to curb the growth of the HIV epidemic. J Infect Dis 198: 59-67.

2. Montaner JS, Hogg R, Wood E, Kerr T, Tyndall M, et al. (2006) The case for expanding access to highly active antiretroviral therapy to curb the growth of the HIV epidemic. Lancet 368: 531.

3. Zachariah R, Teck R, Buhendwa L, Fitzerland M, Labana S, et al. (2007) Community support is associated with better antiretroviral treatment outcomes in a resource-limited rural district in Malawi. Trans R Soc Trop Med Hyg 101: 79-84.

4. Wouters E, van Loon F, van Rensburg D, Meulemans H (2009) Community support and disclosure of HIV serostatus to family members by public-sector antiretroviral treatment patients in the Free State Province of South Africa. AIDS patient care STDs 23: 357-64.

5. Gusdal AK, Obua C, Andualem T, Wahlstrom R, Chalker J, et al. (2011) Peer counselors' role in supporting patients' adherence to ART in Ethiopia and Uganda. AIDS Care 23: 657-62.

6. Zachariah R, Teck R, Buhendwa L, Fitzerland M, Labana S, et al. (2007) Community support is associated with better antiretroviral treatment outcomes in a resource-limited rural district in Malawi. Trans R Soc Trop Med Hyg 101: 79-84.

7. Sanjana P, Torpey K, Schwarzwalder A, Simumba C, Kasonde P, et al. (2009) Task-shifting HIV counselling and testing services in Zambia: the role of lay counsellors. Hum Resour Health 7: 44.

8. Central Statistical Office (CSO), Ministry of Health (MOH) and ICF International. Zambia Demographic and Health Survey 2013-2014. Rockville, Maryland, USA: CSO, Ministry of Health, and ICF International; 2014.

9. Ministry of Health (undated). Adolescent Health Strategic Plan (2011-2015). Ministry of Health, Lusaka, Zambia.

10. World Health Organization (2010) Global experience of community health workers for delivery of health related millennium development goals: a systematic review, country case studies, and recommendations for integration into national health systems. WHO, Geneva.

11. UNAIDS (2012) Global report: UNAIDS report on the global AIDS epidemic: 2012: Geneva.

12. UNICEF (2011) State of the world's children. New York: United Nations Children's Fund.

13. WHO, UNAIDS, UNICEF (2010) Towards universal access: scaling up priority HIV/AIDS interventions in the health sector: progress report 2010. World Health Organization, Geneva.

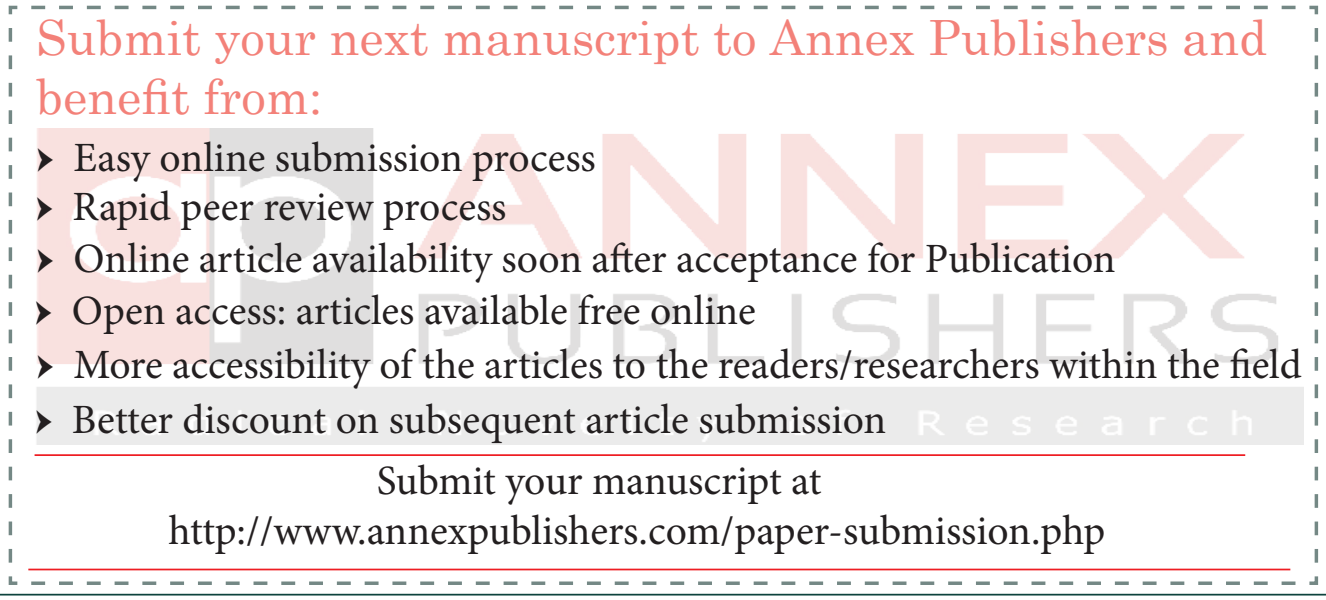

